Philosophical Magazine,

Vol. 00, No. 00, DD Month 200x, 1-6

\title{
Spin-Glass Model for Inverse Freezing
}

\author{
Luca Leuzzi * \\ SMC-INFM/Institute of Complex Systems (ISC) CNR, \\ and Department of Physics, University of Rome, "La Sapienza", Piazzale A. Moro 2, 00185, Rome, Italy \\ (Received 00 Month 200x; in final form 00 Month 200x)
}

We analyze the Blume-Emery-Griffiths model with disordered magnetic interaction displaying the inverse freezing phenomenon. The behaviour of this spin-1 model in crystal field is studied throughout the phase diagram and the transition and spinodal lines for the model are computed using the Full Replica Symmetry Breaking Ansatz that always yelds a thermodynamically stable phase. We compare the results both with the quenched disordered model with Ising spins on lattice gas - where no reentrance takes place - and with the model with generalized spin variables recently introduced by Schupper and Shnerb [Phys. Rev. Lett. 93, 037202 (2004)]. The simplest version of all these models, known as Ghatak-Sherrington model, turns out to hold all the general features characterizing an inverse transition to an amorphous phase, including the right thermodynamic behavior.

\section{Introduction}

In the last years, in a relatively broad number of materials an apparently weird kind of phase transition has been detected and investigated: the "inverse melting". Such a phenomenon was, actually, already hypothesized by Tammann a century ago [1] but it has now been found experimentally in polymeric and colloidal compounds, high- $T_{c}$ superconductors, proteins, ultra-thin films, liquid crystals and metallic alloys [2, 3, 4, 5, 6, 7, 8. This kind of transition, includes, e.g., the solidification of a liquid or the transformation of an amorphous solid into a crystal upon heating. The reason for this counter intuitive process is that a phase usually at higher entropic content happens to exist in very peculiar patterns such that its entropy is decreased below the entropy of the phase usually considered the most ordered one. An example taking place in the widely studied Crystalline Poly(4-methylpentene-1) (P4MP1) 2] is the one of a crystal state of higher entropy that can be transformed into a fluid phase of lower entropy on cooling. Inverse transitions, in their most generic meaning (i.e. both thermodynamic or occurring by means of kinetic arrest), have been observed between fluid and crystal phases [3], between glass and crystal [2] and between fluid and glass ("inverse freezing") 4]. For a comprehensive summary of materials undergoing some kind of phase transition one can turn to Ref. 9]. The aim of this work is to study a simple mean-field model for the inverse transition in a spin-glass, in order to heuristically represent the inverse fluid-amorphous transition.

\section{The Random Blume-Emery-Griffiths-Capel Model}

We have been analyzing the Blume-Emery-Griffiths-Capel (BEGC) model with quenched disorder using the Full Replica Symmetry Breaking (FRSB) scheme of computation that yields the exact stable thermodynamics [11,12,13. It includes the Blume-Capel [14] and the Blume-Emery-Griffiths [15] models, when the couplings are ferromagnetic, and the Ghatak-Sherrington (GS) model, [16] when the couplings are random variables and no biquadratic interaction occurs.

\footnotetext{
* Email: luca.leuzzi@roma1.infn.it 
The model Hamiltonian we consider is

$$
\mathcal{H}=-\sum_{i j} J_{i j} S_{i} S_{j}+D \sum_{i=1}^{N} S_{i}^{2}-\frac{K}{N} \sum_{i<j} S_{i}^{2} S_{j}^{2}
$$

where $S=1,0,-1, D$ is the crystal field, $J_{i j}$ are quenched random variables (Gaussian) of mean zero and variance $1 / N$. The parameter $K$ represents the strength of the biquadratic interaction. A generalized spin variable has been recently proposed [10, 9, for which the degeneracy of the magnetically interacting sites $(S= \pm 1$ ) can be larger (or smaller) than the one of the holes $(S=0)$. To be as inclusive as possible we have been looking at the phase diagram of the random BEGC model also in terms of these generalized variables considering the thermodynamically stable spin-glass obtained by means of the FRSB Ansatz.

Let us call call $k$ the degeneracy of the filled in sites of one type $(S=1$ or $S=-1)$ and $l$ the degeneracy of the empty sites $(S=0)$. The relevant parameter is $r=k / l$. When $r=1$ the spin-1 model is obtained. If, furthermore, $K=0$, the model is the GS one. When, otherwise, $r=1 / 2$ and $D \rightarrow \mu=-D$ the lattice gas formulation of Ref. [17] is recovered, for which no reentrance was observed [11,12].

We discuss the physically stable solution for both the GS model [16, 18, $(K=0)$ and the model with attractive biquadratic interaction [12 $(K / J=1)$, whose phase diagrams are plotted in Fig. 11 and we compare it with the Replica Symmetric (RS) results (see Fig. 2). We study the behaviour of the phase diagram for (a) the lattice gas case $(r=1 / 2)$, for which no inverse transition occurs anywhere in the parameter space; (b) the spin-1 case $(r=1)$, where the first order transition line displays a reentrance soon below the tricritical temperature; (c) the generalized cases as $r>1$, in particular we plot the results of the model with variables taking values $S=\{1,1,0,-1,-1\}$ for which the reentrance takes place above the tricritical point, along the second order phase transition line.

\section{The Full Replica Symmetry Breaking scheme of computation for the Random BEGC model}

Using the replica trick [20] the free energy of $n$ replicas of the system with Hamiltonian Eq. (11) turns out to be

$$
\begin{aligned}
& n \beta f=\frac{\beta}{2}\left(K+\frac{\beta}{2}\right) \sum_{a=1}^{n} \rho_{a}^{2}+\frac{\beta^{4}}{4} \sum_{a \neq b} q_{a b}^{2}-\log \sum_{\{S\}} \exp \left(-\beta \mathcal{H}^{\prime}\right) \\
& -\beta \mathcal{H}^{\prime}=\beta\left(K+\frac{\beta}{2}\right) \sum_{a=1}^{n} \rho_{a} S_{a}^{2}-\beta D \sum_{a=1}^{n} S_{a}^{2}+\frac{\beta^{2}}{2} \sum_{a \neq b} q_{a b}^{2} S_{a} S_{b}
\end{aligned}
$$

where the order parameters introduced in the computation satisfy the self-consistency equations

$$
\rho_{a}=\left\langle S_{a}^{2}\right\rangle \quad q_{a b}=\left\langle S_{a} S_{b}\right\rangle
$$

Choosing the Parisi Ansatz for the matrix $q_{a b}$ and performing infinite steps of RSB [19] the free energy of the spin-glass phase appears to be

$$
\beta f=\frac{\beta}{2}\left(K+\frac{\beta}{2}\right) \rho^{2}-\frac{\beta^{2}}{4} \int_{0}^{1} d x q^{2}(x)-\beta \phi(0,0)
$$

and the self-consistency equations become

$$
q(x)=\int_{-\infty}^{\infty} d y P(x, y) \phi^{\prime}(x, y)^{2} \quad \rho=\int_{-\infty}^{\infty} d y P(1, y) \frac{2 r e^{\Theta} \cosh \beta y}{1+2 r e^{\Theta} \cosh \beta y}
$$



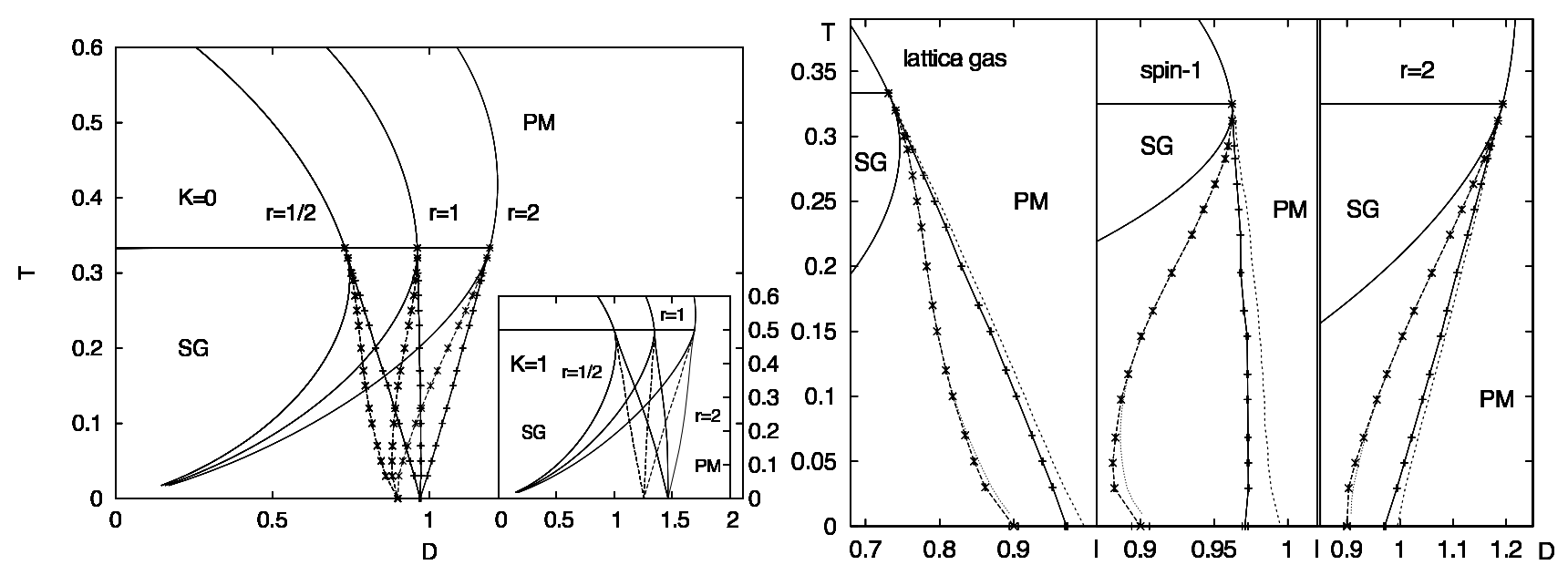

Figure 1. The $D-T$ phase diagram in absence of biquadratic interaction. Three models with different behaviors are plotted: $r=1 / 2,1$, 2. For each $r$-model three curves are represented, each departing from the same tricritical point: the full curve on the left is the spinodal of the PM phase, the dashed one in the middle is the first order transition line and the right one is the SG spinodal line. The group of three curves on the left are for the Ising spins on lattice gas $\left(r=1 / 2, T_{c}=1 / 3, D_{c}=0.73105\right)$. The group of curves in the middle represent the lines of the GS model $\left(r=1, D_{c}=0.96210\right)$. The curves on the right correspond to the $r=2$ model $\left(D_{c}=1.19315\right)$. In the inset the same diagram is plotted when $K=1$ (attractive biquadratic interaction). No qualitative difference occurs.

Figure 2. $\quad D$ - $T$ diagrams at $K=0$ : Ising spin glass on lattice gas (left), GS model (center) and random BEGC model with $r=2$ (right). Both the RS and the FRSB solution (the latter with error bars) are plotted. For $r=1 / 2$ no reentrance occurs. A reentrance occurs, instead, below the tricritical point in the GS model along the first order transition line (and a second reentrance seems is there for $T<0.03)$. In the latter model the reentrance occurs above the tricritical point.

where $\Theta \equiv \beta^{2} / 2[\rho-q(1)]+\beta K \rho-\beta D$. The functions $\phi(x, y)$ and $P(x, y)$ are solutions of the non-linear partial differential equations

$$
\begin{aligned}
& \dot{\phi}(x, y)=-\frac{\dot{q}}{2}\left\{\phi^{\prime \prime}(x, y)+\beta x\left[\phi^{\prime}(x, y)\right]^{2}\right\} \\
& \dot{P}(x, y)=-\frac{\dot{q}}{2}\left\{P^{\prime \prime}(x, y)-2 \beta x\left[P(x, y) \phi^{\prime}(x, y)\right]^{\prime}\right\}
\end{aligned}
$$

with boundary conditions

$$
\phi(1, y)=\frac{1}{\beta} \log \left(2+4 r e^{\Theta} \cosh \beta y\right) \quad P(0, y)=\delta(y)
$$

The dot and the apex respectively represent the derivative with respect to $x$ and $y$. For a detailed study of the above equations the reader can look at Ref. [12.

Having a model with variables displaying a relative degeneracy $r\left(D=D_{r}\right)$, in order to describe the partition function of another model whose variables have degeneracy $r^{\prime}$ it is enough to vary the crystal field as $D_{r^{\prime}}+T \log r^{\prime}=D_{r}+T \log r$. This does not hold, however, for the state functions obtained deriving the thermodynamic potential with respect to the temperature (e.g. entropy and internal energy) that will, instead, receive contributions from additional terms. For instance, the entropy function $(K=0)$ is

$$
\begin{aligned}
s(\beta, D)= & -\frac{\beta^{2}}{4}[\rho-q(1)]^{2}-\frac{\beta^{2}}{2} \rho[\rho-q(1)]-\beta \rho D+\rho \log (2 r)+\beta^{2} \int_{0}^{1} d x q^{2}(x) \\
& +\int_{-\infty}^{\infty} d y P(1, y) \log \{2+4 \exp (\Theta+\log r) \cosh \beta y\}
\end{aligned}
$$

Identifying $D_{1 / 2} \equiv-\mu$ in Eq (10) one recovers the case of spins on a lattice gas of chemical potential $\mu 11$. 


\section{Thermodynamics}

By solving Eqs. (5)-(10) we build the $D-T$ phase diagrams that we plot for $K=0$ and $K=1$ in Fig. 1 and inset, at different values of $r$. The reentrance in the $D$ - $T$ plane is present already in the spin-1 GS model. As a consequence this implies that there is no need for the intuition of Ref. [10] in order to have a model for inverse freezing from low temperature liquid to high temperature amorphous solid. This is at difference with the liquid-crystal inverse transition ("inverse melting") for the description of which the original Blume-Capel model is not adequate and $r>1$ is needed [10,9].

The transition lines are not very much dependent on the Ansatz used to compute the quenched average of the free energy. For not extremely low $T$, the first order transition lines yielded by the RS and the FRSB Ansatz even coincide down to the precision of our numerical evaluation of the FRSB antiparabolic Parisi equation (7) whereas they slightly differ at very low temperature (see inset of Fig. 2). For what concerns the spinodal lines, the RS ones are shifted inside the pure paramagnetic (PM) phase.

Introducing a biquadratic interaction term and varying it from attractive to repulsive the situation does not change much (see e.g. inset of Fig. 11). For any value of $K$ no reentrance of the phase transition line occurs in the $D-T$ phase diagram of the lattice gas model whereas it is always there for the spin- 1 model. The only consequence of reducing $K$ is that the area of the phase coexistence region is reduced (the tricritical temperature tends to zero as $K \rightarrow-\infty)$.

The slope of a first order line is given by the Clausius-Clapeyron equation. For the BEGC model it can be written in terms of the crystal field $D$ (playing the role of a chemical potential), instead of the pressure that is not defined in our model: ${ }^{1}$

$$
\frac{d D}{d T}=\frac{s_{\mathrm{pm}}-s_{\mathrm{sg}}}{\rho_{\mathrm{pm}}-\rho_{\mathrm{sg}}}=\frac{\Delta s}{\Delta \rho}
$$

This formula is valid for any $r$. We stress that in passing from $r$ to $r^{\prime}$ also the entropy (Eq. 10) changes of a term $\rho \log r / r^{\prime}$, in agreement with the crystal field shift given above.

In Fig. 4 the behaviour of the entropy as a function of the temperature (Eq. (10)) is shown across an inverse transition (as a function of the crystal field $D$ in the inset) for the spin-1 model. The entropy of the PM phase below the first order transition line is smaller than the entropy of the SG: heating the system the paramagnet becomes an amorphous magnet (i.e. "freezes") acquiring latent heat from the heat bath. Going down along the transition line, as $\Delta s$ changes sign in Eq. (11) the slope becomes positive. The $\Delta s=0$ point is called a Kauzmann locus 3 .

A reentrance in the transition line can be due both to the existence of a liquid phase with an entropy lower than the one of the solid or to a the liquid phase more dense than the solid one (like in the water-ice transition). When an entropic inversion accounts for the phase transition, the equilibrium transition line changes slope in a point where the entropy of the fluid phase, $s_{\text {liq }}$, becomes equal to the one of the solid, $s_{\text {sol }}$, according to the Clausius Clapeyron equation for first order phase transitions. From this point on a whole iso-entropic line, $\Delta s=s_{\text {liq }}-s_{\text {sol }}=0$, can be continued both inside the solid and the liquid phases. This is a particularly interesting observation since, in the context of glass formers, a transition to an "ideal" glass at the temperature at which $\Delta s=0$ is hypothesized, in order to avoid the paradox that an under-cooled liquid might possess less entropy than the associated crystal at the same values of the thermodynamic parameters 21]. From an experimental point of view this Kauzmann temperature would be the temperature of the glass transition (that is not a true phase transition because strictly kinetic in origin) in an idealized adiabatic cooling procedure. Since the astronomically long relaxation time needed to actually perform such an experiment makes such a procedure unfeasible, the evidence in favour of the existence of a thermodynamic glass transition mainly comes from analytical and numerical investigations (see e.g. Refs. 22,23]). The fact that a $\Delta s=0$ line turns out naturally in the description of the behavior of materials with inverse transition avoids, at least for these substances, the Kauzmann paradox and breaks the connection between $\Delta s=0$ extrapolation and the existence of an ideal amorphous phase [3,2].

\footnotetext{
${ }^{1}$ With respect to the classic Clausius-Clapeyron equation $D$ takes the place of the pressure and $\rho$ plays the role of the specific volume.
} 

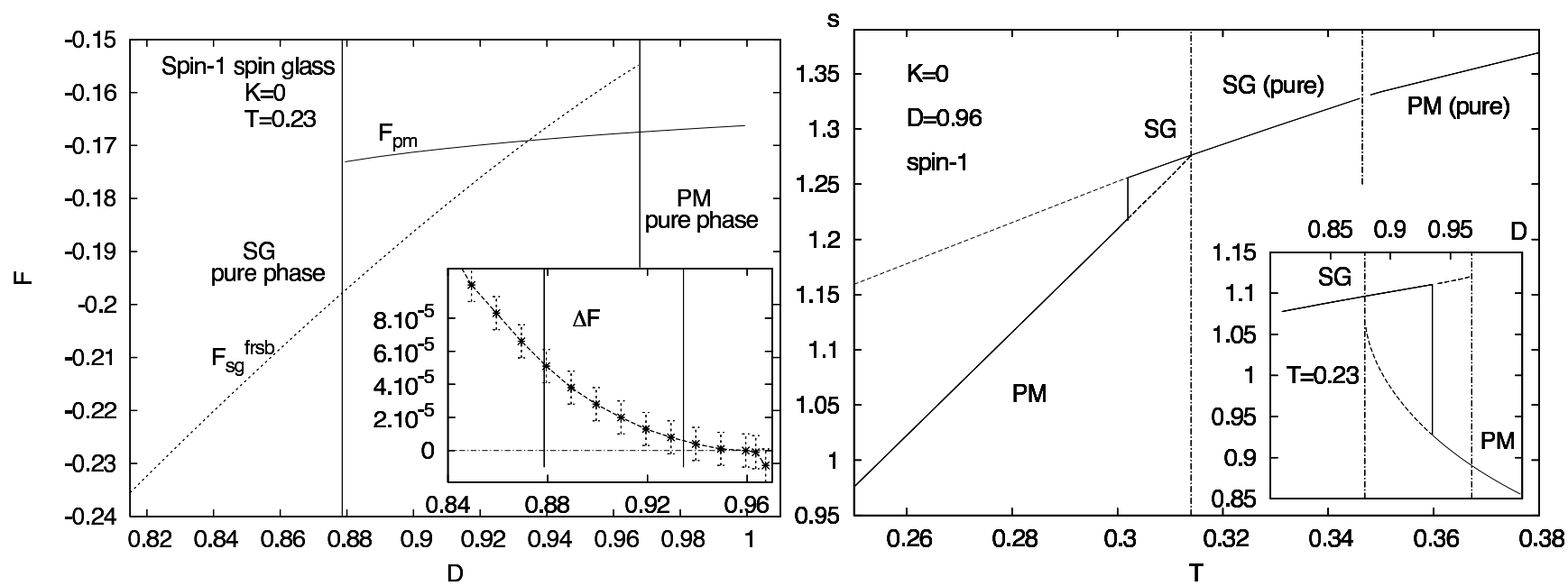

Figure 3. Free energy $F_{\mathrm{pm}}$ and $F_{\mathrm{sg}}^{\mathrm{frsb}}$ vs. $D$ at $T=0.23$ in the GS model $(K=0)$. The left and right side vertical lines are at the spinodal points of, respectively, the PM and the SG phase. The first order transition occurs at $D_{1} s t=0.9344$. In the inset $\Delta F=F_{\mathrm{sg}}^{\mathrm{rrsb}}-F_{\mathrm{sg}}^{\mathrm{rs}}$ is displayed. At this temperature the two functions merge very near to the tricritical point (right side vertical line).

Figure 4. Entropy vs. $T$ at the crystal field value of $D=0.96$ for the GS model. $T_{1} s t=0.302$. The two vertical dashed lines are the SG $\left(T_{\mathrm{SG}}=0.314\right)$ and $\mathrm{PM}\left(T_{\mathrm{PM}}=0.3465\right)$ spinodal lines. In the inset $s(D)$ is plotted at $T=0.23$.

\section{Conclusions}

We have shown that the Ghatak-Sherrington model undergoes the inverse freezing phenomenon acquiring latent heat from the heat bath as the paramagnet becomes a spin-glass. Many other models can be built starting from this one, introducing an attractive or repulsive biquadratic interaction (the last term in Hamiltonian (10) and/or tuning the relative degeneracy of the value $S=0$ and $S^{2}=1$ of the spin variable (as in [10]) but the GS one already contains all the features needed to qualitatively represent the experimental results.

We have considered here a case where the frozen phase is a spin-glass and the transition is only static: in order to have a structural glass and to deal with a dynamic (mode coupling) transition, it would be, however, enough to apply the simpler one step RSB Ansatz in, e.g., a model with a $p$-body magnetic interaction (with $p>2$ instead of a couple interaction), such as the one introduced in Ref. 24] or an analytically solvable model with spherical variables instead of discrete ones and again a multi-body interaction (a valuable starting point would be the work of Ref. 25]). Else, a spin-1 model with an orthogonal matrix $J_{i j}$, instead of the Gaussian one, can be used, as latterly done by Sellitto [26. Besides the inverse freezing phenomenon this last model also displays a fluid-fluid transition (between two distinct paramagnetic phases), a feature recently found in the solution of 4-methil-piridyne (4MP) and $\alpha$-cyclodextrin in water, investigated by Angelini and coworkers [8].

With the help of the class of models presented here the connection between entropy driven phase reentrance and shear thickening can also be tackled [27] and, furthermore, a generalization of the spin-1 variable to a composition of "fast" and "slow" variables ${ }^{1}$ coupled to two different thermal baths allows for studying anomalous latent heat in out of equilibrium transitions [28].

\section{References}

[1] G. Tammann, "Kristallisieren und Schmelzen", Metzger und Wittig, Leipzig (1903).

[2] S. Rastogi, G.W.H. Höhne and A. Keller, Macromolecules 32, 8897 (1999); A.L. Greer, Nature 404, 134 (2000); N.J.L. van Ruth and S. Rastogi, Macromolecules 37, 8191 (2004).

[3] F.H. Stillinger, P.G. Debenedetti and T.M. Truskett, J. Phys. Chem. B 105 (2001), 11809;

F.H Stillinger and P.G. Debenedetti, Biophys. Chem. 105 (2003) 211.

[4] C. Chevillard and M.A.V. Axelos, Colloid. Polym. Sci. 275, 537 (1997).

[5] O. Portman, A. Vaterlaus and D. Pescia, Nature 422, 701 (2003).

[6] M. Takahashi, SHimazaki and Yamamoto, J. Polym. Sci. 39, 91 (2001)

\footnotetext{
${ }^{1}$ E.g., setting $S=\sigma n$, with $n=0,1$ fast and $\sigma= \pm 1$ slow
} 
[7] M. Plazanet et al., J. Chem. Phys. 121, 5031 (2003)

[8] R. Angelini, this volume.

[9] N. Schupper and N.M. Shnerb, Phys. Rev. E 72, 046107 (2005).

[10] N. Schupper and N.M. Shnerb, Phys. Rev. Lett. 93 (2004) 037202.

[11] A. Crisanti and L. Leuzzi, Phys. Rev. Lett. 89 (2002) 237204.

[12] A. Crisanti and L. Leuzzi, Phys. Rev. B 70 (2004) 014409.

[13] A. Crisanti and L. Leuzzi, Phys. Rev. Lett. 95, 087201 (2005).

[14] H. W. Capel, Physica 32(1966) 966; M. Blume, Phys. Rev. 141 (1966) 517.

[15] M. Blume, V.J. Emery and R.B. Griffiths, Phys. Rev. A 4 (1971) 1071.

[16] S. K. Ghatak, D. Sherrington, J. Phys. C: Solid State Phys. 10, 3149 (1977).

[17] J.J. Arenzon, M. Nicodemi and M. Sellitto, J. Phys. I France 6 (1996) 1143.

M. Sellitto, M. Nicodemi, J.J. Arenzon, J. Phys. I (France) 7 (1997) 945.

[18] P.J. Mottishaw, D. Sherrington, J. Phys. C: Solid State Phys. 18, 5201 (1985).

[19] G. Parisi, J. Phys. A 13, L115 (1980).

[20] M. Mezard, G. Parisi and M. Virasoro, (1986).

[21] W. Kauzmann, Chem. Rev. 43, 219 (1948).

[22] M. Mezard, Physica A 265, 352 (1999); M. Mezard and G. Parisi, J. Chem. Phys. 111, 1076 (1999).

[23] B. Coluzzi, G. Parisi and P.Verrocchio, J. Chem. Phys. 112, 2933 (2000); Phys. Rev. Lett. 84, 306 (2000).

[24] P.J. Mottishaw, Europhys. Lett. 1, 409 (1986)

[25] A. Caiazzo, A. Coniglio and M. Nicodemi, Phys. Rev. Lett. 93, 215701 (2004).

[26] M. Sellitto, cond-mat/0603381

[27] M. Sellitto and J. Kurchan, Phys. Rev. Lett. 95, 236001 (2005).

[28] A. Allahverdyan and Petrosyan, Phys. Rev. Lett. 96, 065701 (2006). 